\title{
2-R REGENERATION EXPLOITING SELF-PHASE MODULATION IN A SEMICONDUCTOR OPTICAL AMPLIFIER
}

\author{
Gianluca Meloni, ${ }^{1}$ Antonella Bogoni, ${ }^{2}$ and Luca Poti ${ }^{2}$ \\ ${ }^{1}$ Scuola Superiore Sant'Anna, P.zza dei Martiri della Libertà 33, 56100 Pisa \\ ${ }^{2}$ CNIT, Via Cisanello 145, 56124 Pisa;
}

\begin{abstract}
All-Optical Re-amplification and Re-shaping (2R) exploiting spectral shift induced by Self-Phase-Modulation (SPM) in a Semiconductor Optical Amplifier (SOA) is presented. Theoretical model taking into account Carrier Density (CD), Carrier Heating $(\mathrm{CH})$ and Spectral Hole Burning (SHB) is experimentally validated using 3 ps optical pulses.
\end{abstract}

\section{INTRODUCTION}

The increase of transmission bandwidth request in the optical communications and networks opens new research perspective towards ultra-fast alloptical systems. Optical Time Division Multiplexed (OTDM) systems together with all-optical packet switching networks represent the future. However, ultra short signals are strongly affected by different kind of noise sources as, for example, Polarization Mode Dispersion, Chromatic Dispersion, Nonlinear fiber effects, Amplified Spontaneous Emission, etc... In this contest opto electronic conversion, regeneration, and electro-optic conversion, are unthinkable. The electrical bottleneck must be overcame exploring all-optical regeneration techniques.

In this paper, we propose a new technique to perform $2 \mathrm{R}$ (Re-shaping and Re-amplification) all-optical regeneration using a Semiconductor Optical Amplifier (SOA) and a Tunable Optical Filter (TOF). The principle exploits the phenomenon of Self Phase Modulation into the SOA. SOA can be a key device for such applications in future optical networks because of its integrability, nonlinear efficiency, low cost and reliability. 
The paper describes the model used to numerically investigate the phenomenon. An experimental validation of the results are also reported.

\subsection{R-Regenerator Basic Principle}

$2 \mathrm{R}$ Regeneration stands for Re-amplification and Re-shaping. Former functionality is easily obtained, in the optical domain, using Fiber, Semiconductor or Raman amplifiers. However, amplification is not always sufficient to guarantee good performance for high capacity transmission systems, where Re-shaping can be introduced. The principle of operation for the re-shaper is based on the ideal characteristic shown in Fig.1 where the output power is plotted as a function of the input power.

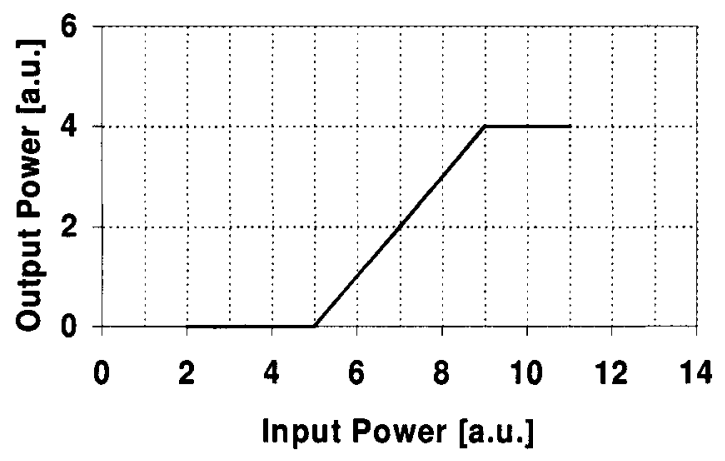

Figure 1. Re-shaper ideal characteristic.

The input/output characteristic represents a typical soft limiter transfer function. In this way, if the re-shaper signal input has power fluctuations, due to any kind of noise accumulated during its propagation, they can be partially or completely eliminated by the limiter nonlinear characteristic. The effect can be exploited both on the space and mark levels. An ideal 2R-Regeneration, hence, is a nonlinear amplifier that, at the same time, properly shapes and amplifies the input signal. For this purpose, we exploit Self-Phase Modulation (SPM) in a SOA. When a powerful signal propagates into a SOA, it experiences a nonlinear phase modulation (SPM) induced by a refractive index variation due to carrier change in the conduction bandwidth. As the carrier change is governed by fast phenomena (Carrier Density - $\mathrm{CD}$, Carrier Heating - $\mathrm{CH}$, and Spectral Hole Burning - SHB), the phase modulation exactly follows the peak power time evolution. When a powerful pulsed or modulated Return-to-Zero (RZ) signal propagates through a SOA, its phase is modulated, thus giving an optical spectrum broadening and red shift. In other words, an high mark level at the SOA output is wavelength-shifted in respect to a space level. The wavelength 
shift is proportional to the input peak power. It is possible, than, to use an optical filter centered on the shifted signal to eliminate the noise on the space level and to limit the power fluctuation on the mark level.

\subsection{Self-Phase Modulation model for SOAs}

In [1] the nonlinear Kerr effect (refractive index modulation) is included in an accurate model and is described by the dependence of the effective refractive index $n$ on intensity $I$ :

$$
n(\omega, I)=n_{0}(\omega)+n_{2} I
$$

where $n_{0}$ is the linear index, $n_{2}$ is the Kerr coefficient ${ }^{1}$, and $\omega$ the pulsation. When the intensity $I(t)$ is modulated, the refractive index changes in time, and the propagation constant becomes:

$$
\beta(\omega, I)=\frac{\omega n(\omega, I)}{c}
$$

The study of the medium response to the optical field leads to the following set of three equations:

$$
\begin{gathered}
\frac{\delta P}{\delta z}=\left(g-\alpha_{i n t}\right) P \\
\frac{\delta \Phi}{\delta z}=-\frac{1}{2} \alpha g \\
\frac{\delta g}{\delta \tau}=\frac{g_{0}-g}{\tau_{c}}-\frac{g P}{E_{s a t}}
\end{gathered}
$$

where $\alpha_{\text {int }}$ is the internal loss, $E_{\text {sat }}$ is the saturation energy of the amplifier, $P(z)$ and $\Phi(z)$ are the power and the phase, $g_{0}$ is the small signal gain, $\tau_{c}$ is the spontaneous carrier lifetime, $\alpha$ is the line-width enhancement factor ( [2], [3]), and $g$ is the saturated gain. Equation 4 shows the origin of SPM. Carrier population dynamics must considered in the model to properly account for ultra-fast intra- and inter- band phenomena:

- Carrier Density (inter-band phenomenon)

- Spectral Hole Burning (intra-band phenomenon)

- Carrier Heating (intra-band phenomenon).

Inter band means that carrier are exchanged between valence and conduction bandwidths, whereas intra band includes those phenomena that are generated by a carrier displacement in the same bandwidth. The former have time constants of about $100 \mathrm{ps,} \mathrm{and} \mathrm{the} \mathrm{latter} \mathrm{three} \mathrm{order} \mathrm{of} \mathrm{magnitude} \mathrm{lower.}$

$\overline{{ }^{1} n_{2} \text { is approximately } 3.2 \times 10^{-16} \mathrm{~cm}^{2} / W}$ 
The intra/inter-band phenomena are included in the model by considering a gain:

$$
g(t)=\overbrace{\frac{\overbrace{N}(t)}{\underbrace{f(t)}_{\text {SHB }}}}^{\mathbf{C D}}+\overbrace{\Delta g_{T}(t)}^{\mathbf{C H}}
$$

where $g_{N}(t), \Delta g_{T}(t)$, and $f(t)$ represent $\mathrm{CD}, \mathrm{CH}$, and SHB respectively.

\section{NUMERICAL RESULTS}

When an ultra-short optical pulse propagates through a SOA, the gain quickly saturates, due to the carrier consumption on the conduction bandwidth, and afterward it recovers with time constants that are strictly related to the intra/inter band phenomena. As an example, Fig. 2 shows the SOA gain time saturation and recovery when a 1.2 ps long pulse crosses the device. Few carriers are moved within the conduction bandwidth very quickly by $\mathrm{CH}$ and SHB giving the first fast gain recovery, whereas most of the bandwidth is repopulated by the bias current $(\mathrm{CD})$.

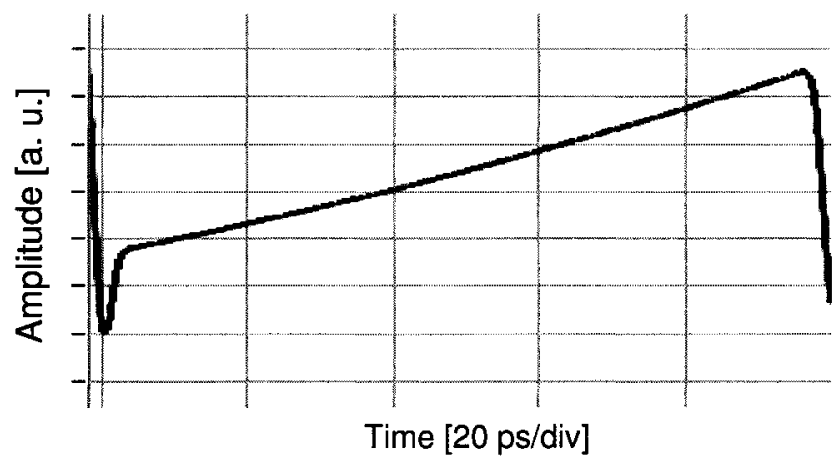

Figure 2. Gain dynamic.

The model is here used, than, to simulate for the spectrum red shift. In Fig. 3 is plotted the optical spectrum of the signal at the input (line) and output (dashed-line) of the SOA and at the output (dotted-line) of the Tunable Optical Filter (TOF) after the amplifier.

First we simulate a $15 \mathrm{ps}$ clock signal regeneration. A $10 \mathrm{GHz}$ pulsed signal at $1548.7 \mathrm{~nm}$ is propagated through a SOA and the output is selected using a TOF centered around $1550 \mathrm{~nm}$ with a bandwidth of $1 \mathrm{~nm}$. The time signals are shown in Figg.4(a) and 4(b) 


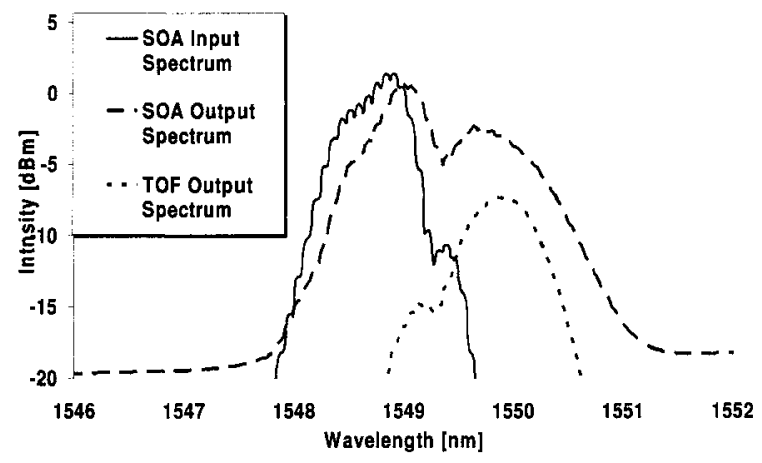

Figure 3. Simulated Spectra at the SOA input (line), output (dashed-ine), and after the TOF (dotted-line)

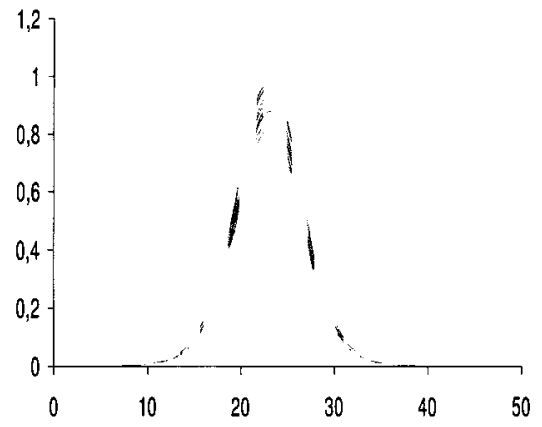

(a)

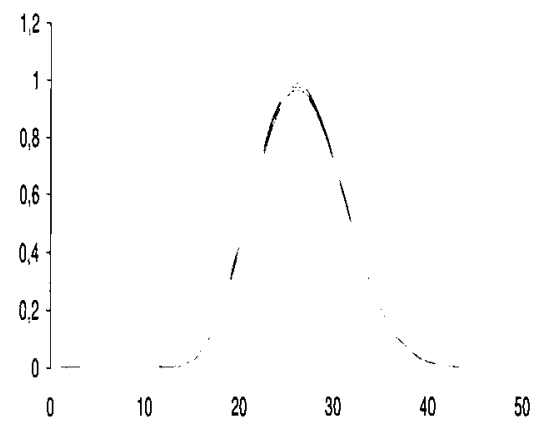

(b)

Figure 4. (a) Input pulses; (b) Output regenerated pulses

Numerical results shows a sensible noise reduction on the peak power. An input peak change of about $\pm 10 \%$ around the mean level is reduced to less than $\pm 2 \%$ at the TOF output.

As a second case study, a modulated signal, with same parameter as before, has been simulated. The considered sequence is ...10101010... where the optical modulator extinction ratio is $20 \mathrm{~dB}$. Input and output eye diagrams are shown in Fig.5.

Also in this case we may appreciate an improve of about $\pm 3 \%$ on the mark level, and a small noise reduction also on the space level. 


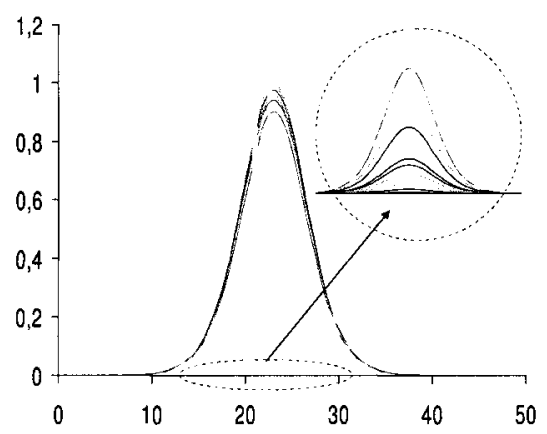

(a)

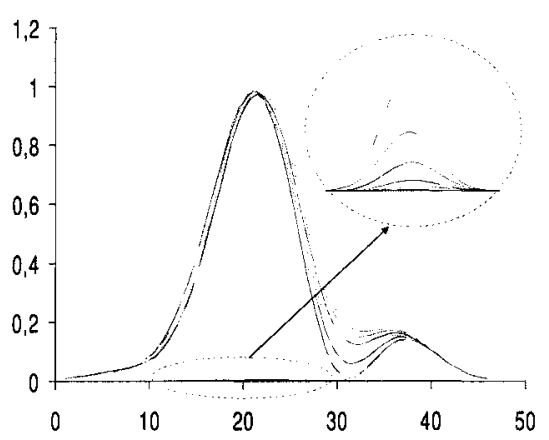

(b)

Figure 5. (a) Input eye diagram; (b) Output eye diagram

\section{EXPERIMENTAL ACTIVITY}

The experimental setup is shown in Fig.6. A Mode-Locked Fiber Laser (MLFL) generates $20 \mathrm{ps}$ optical transform-limited pulses at $10 \mathrm{GHz}$. A following compression stage composed by higher order soliton generator and a pedestal suppressor is used to reduce the time duration down to $2 \mathrm{ps}$. The optical pulses are than amplified and modulated using a Mach-Zehender electrooptic modulator. Two isolators are used at both the SOA ports to avoid any reflection and lasing, and a polarization controller assures maximum nonlinear efficiency. The SOA output signal is than filtered using a $1 \mathrm{~nm}$ bandwidth TOF. The signal is than measured by an Optical Spectrum Analyzer (OSA) and a 50

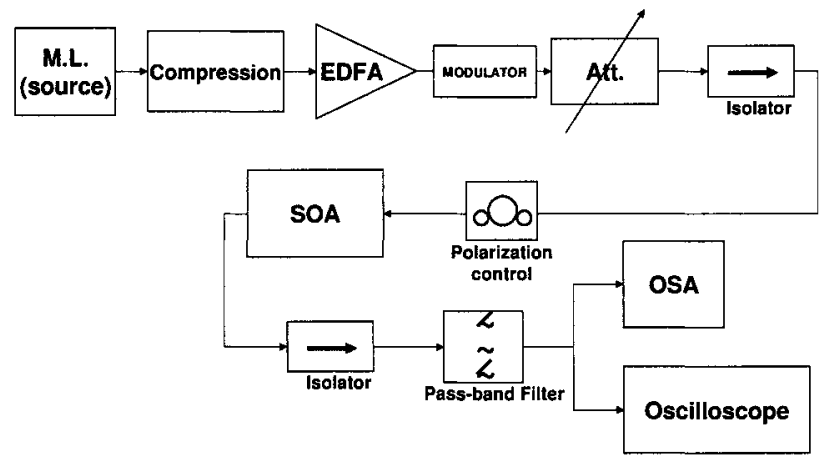

Figure 6. Experimental Set-up.

$\mathrm{GHz}$ bandwidth sampling oscilloscope. Fig. 7 shows the measured specrta at the SOA input, output and after the TOF. The red shift is clear from the graph. 


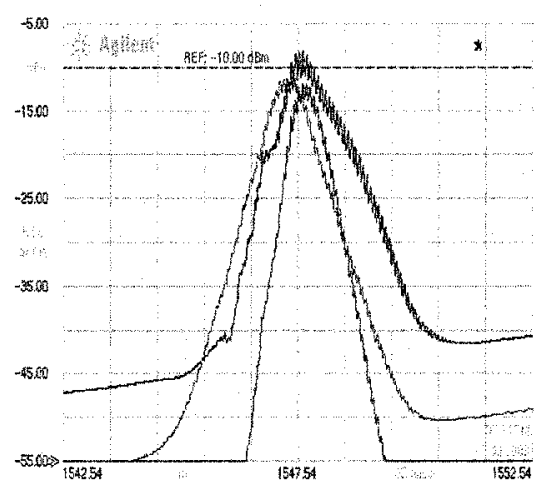

Figure 7. Measured spectra with an input power of $5 \mathrm{dBm}$

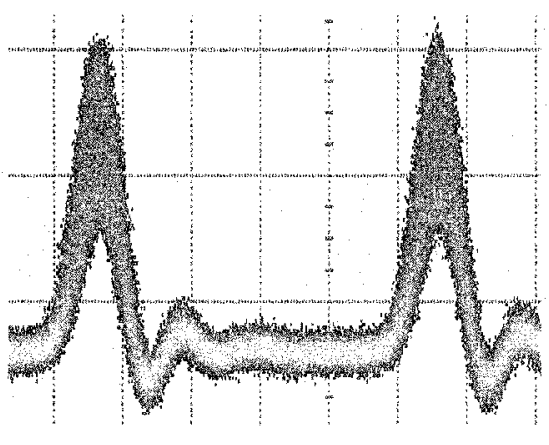

(a)

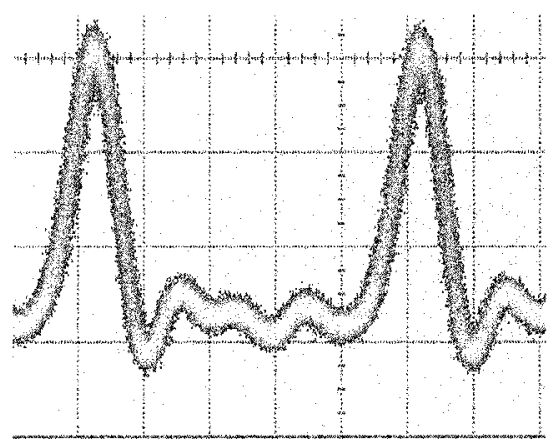

(b)

Figure 8. (a) Input distorted pulses; (b) Output regenerated pulses

The measured pulses time trace are shown in Fig. 8 when an input mean power of $5 \mathrm{dBm}$ is used. By increasing the compressor power it is possible to increase both the input SOA power and the pulse distortion. Fig.9 shows the distorted input (left) and regenerated output (right) pulses for an input power of about $8 \mathrm{dBm}$. The reshaping effect, in this case, is more evident.

\section{CONCLUSION}

In this paper we have theoretically demonstrated and experimentally implemented a $2 \mathrm{R}$ regenerator. Nonlinear reshaping characteristic is obtained exploiting SPM in SOA and following spectral filtering. The scheme has been successfully implemented using $10 \mathrm{GHz}$ optical transform-limited pulses with time duration lower than $3 \mathrm{ps}$. 


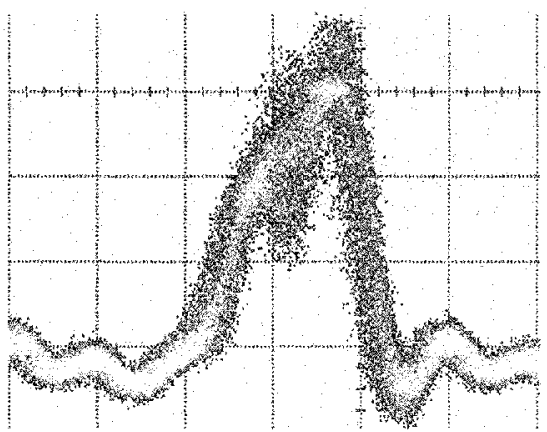

(a)

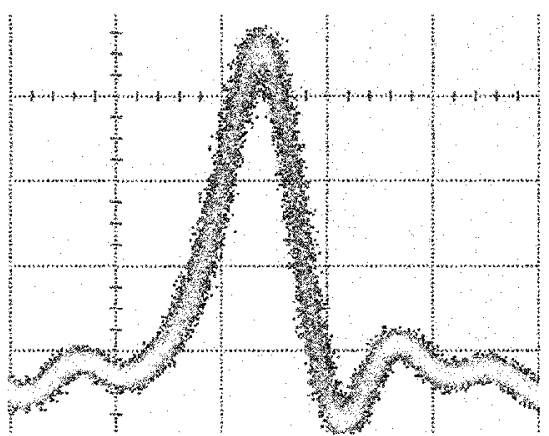

(b)

Figure 9. (a) Input distorted pulses; (b) Output regenerated pulses

\section{REFERENCES}

[1] M.Stern, J.P.Heritage, R.N.Thurston, and S.Tu, "Self-phase modulation and dispersion in high data rate fiber-optic transmission systems," Journal of Lightwave Technology, vol. 8, july 1990.

[2] C.H.Henry, "Theory of the linewidth of semiconductor lasers," IEEE J. Quantum Electron., vol. QE-18, pp. 259-264, 1982.

[3] M.Osinski and J.Buus, "Linewidth broadening factor in semiconductor lasers-an overview," IEEE J. Quantum Electron., vol. QE-23, pp. 9-29, 1987.

[4] A.Bizzi, "Sviluppo di un simulatore di amplificatori ottici (soa) e suo impiego nella realizzazione di un modulatore ottico ultraveloce per sistemi otdm," 2000-2001. Thesis. 